\title{
Étude électromyographique paravertébrale dorso-Iombaire Analyse en mode multi-MUP et établissement de normes au sein d'une population de référence
}

\author{
Marco Tomasella*, Jean-Michel Crielaard, François-Charles Wang \\ Département d'Électroneuromyographie, CHU Sart Tilman, 4000 Liège, Belgique \\ (Reçu le 30 juillet 2001 ; accepté le 18 janvier 2002)
}

\begin{abstract}
Résumé - Ce travail vise à établir des normes pour les paramètres électromyographiques de la musculature paravertébrale dorso-lombaire.
\end{abstract}

Matériel et Méthodes. 75 sujets sains, asymptomatiques sur le plan rachidien, sont soumis à une étude électromyographique des faisceaux musculaires du multifidus dont l'innervation monosegmentaire dépend du rameau postérieur du nerf rachidien. Les myotomes T8 , L3 , L4 , L5 et S1 sont explorés. L'activité spontanée de repos et les paramètres quantitatifs des potentiels d'unité motrice obtenus par l'analyse en mode multi-MUP (Motor Unit Potential) sont étudiés.

Résultats et conclusion. Aucun potentiel de fibrillation, aucun potentiel lent de dénervation ni aucune fasciculation n'est enregistré au repos. II n'apparaît aucune influence du sexe ou de l'âge sur l'analyse quantitative des potentiels d'unité motrice. Des normes de référence sont établies pour les niveaux segmentaires T8 et $L 5$ sur le calcul des moyennes des 20 potentiels d'unité motrice isolés chez chaque sujet. Un intervalle de référence est également défini à partir des limites extrêmes inférieure et supérieure des valeurs individuelles. Cette étude met à la disposition des électrophysiologistes des valeurs de référence permettant d'objectiver une éventuelle pathologie myogène ou neurogène, en particulier dans le cadres des radiculopathies lombo-sacrées. (c) 2002 Éditions scientifiques et médicales Elsevier SAS

électromyographie / muscles paravertébraux / analyse EMG quantitative / potentiel d'unité motrice / valeurs de référence.

Summary - Dorsal and lumbar paraspinal electromyographic study. Multi-MUP analysis and drawing up normal values in a reference population. Objective. The aim of this study was to contribute to draw up reference values relating to electromyographic (EMG) parameters in dorsal and lumbar paraspinal muscles.

Materials and Methods. 75 healthy subjects without back pain underwent electromyography of multifidus bundles, which are innervated uni-segmentally by the dorsal ram of the spinal nerve. T8, L3, L4, L5 and S1 myotomes were systematically explored. Output variables were spontaneous denervation activity and quantitative EMG data obtained by multi-MUP (Motor Unit action Potential) analysis.

* Correspondance et tires à part.

Adresse e-mail : m.tomasella@chu.ulg.ac.be (M. Tomasella). 
Results. No abnormal insertional or spontaneous activity (such as fibrillation, positive sharp waves or fasciculation) was recorded at rest. Neither sex nor age influenced motor unit action potential features in our series. Reference values were drawn up for $\mathrm{T} 8$ and $\mathrm{L} 5$ segmental levels using the mean values of 20 motor unit potentials in each patient studied, the reference interval being defined by the lower and the upper outlier limits on individual values.

Conclusion. This study offers reference data to electromyographers to help better identifying possible myogenic or neurogenic pathological changes, especially in lumbosacral radiculopathies. (c) 2002 Éditions scientifiques et médicales Elsevier SAS

electromyography / paraspinal muscles / quantitative EMG analysis / motor unit potential / reference values.

\section{INTRODUCTION}

Les radiculopathies sciatiques ou crurales peuvent s'observer lors d'un conflit disco- ou ostéophytoradiculaire. On rappellera également, dans la genèse de la lombo-sciatique, l'importance des médiateurs chimiques du processus inflammatoire susceptibles d'engendrer diverses altérations neurophysiologiques de la racine nerveuse, même lors d'un contact sans compression [4].

Malgré un contexte clinique évocateur et une imagerie médicale démonstrative, de nombreuses investigations électrophysiologiques restent normales et n'apportent pas la preuve du conflit radiculaire.

Depuis une dizaine d'années, la littérature scientifique se fait l'écho d'un regain d'intérêt pour l'examen électromyographique de la musculature paravertébrale dans le cadre des radiculopathies $[1,5,8,11,15,19]$. Cet examen permet de recueillir au niveau des muscles paravertébraux des signes précoces ou chroniques de dénervation, ciblant davantage le niveau segmentaire de l'atteinte radiculaire $[1,5,15]$.

L'objectif de ce travail sera d'établir des normes, relatives à l'exploration électromyographique des muscles paravertébraux.

\section{RAPPEL ANATOMIQUE}

Les travaux de dissection de Bogduk et al. et de Mac Intosch et al. ont défini les modalités d'insertion des différents groupes musculaires paravertébraux $[3,14]$. La masse commune des muscles érecteurs du tronc se compose, respectivement de dedans en dehors, du multifidus, du longissimus et de l'iliocostalis.

Le multifidus lombaire se dispose segmentairement selon un schéma d'insertion spino-transverse. Chaque faisceau musculaire comporte 5 fascicules, distinctement individualisés par un plan de clivage, s'insérant proximalement sur la lame osseuse, le rebord inféro- latéral et le sommet d'une apophyse épineuse pour s'échelonner caudalement et latéralement sur les tubercules postérieurs des apophyses transverses des 2ème, 3ème et 4ème vertèbres sous-jacentes [8]. L'innervation de cette musculature paravertébrale dépend du rameau postérieur du nerf rachidien. Soulignons les rapports anatomiques étroits existant entre ce rameau postérieur et l'articulation zygapophysaire qu'il contourne avant d'innerver les muscles périrachidiens [3]. Le multifidus bénéficie d'une innervation monosegmentaire particulière: chaque fascicule, inséré sur le sommet d'une apophyse épineuse, est innervé spécifiquement par le rameau postérieur provenant d'un même niveau segmentaire $[3,8,9,13,18]$. Cette innervation monosegmentaire caractérise également le muscle interépineux $[3,8,18]$. Bogduk n'a pas confirmé l'existence de fibres musculaires appartenant au multifidus sur l'apophyse épineuse de $S 1$ : l'innervation monosegmentaire du multifidus en $S 1$ pourrait être remise en question [3, 10].

L'analyse de la disposition transversale des muscles rachidiens montre que l'accolement et l'innervation des faisceaux musculaires multifides provenant de niveaux segmentaires différents s'établissent spécifiquement de dedans en dehors [18] : en regard du niveau segmentaire L4-L5, les faisceaux les plus internes par rapport au plan de l'apophyse épineuse sont innervés par le rameau postérieur de L4, ceux en position intermédiaire par le rameau postérieur de L3 et les plus externes font partie du myotome L2. La recherche du contact osseux de l'aiguille électromyographique (EMG) au niveau de l'apophyse épineuse permet d'affirmer le positionnement adéquat de celle-ci en fonction de l'innervation recherchée [18].

Par contre, les faisceaux musculaires intermédiaires $\mathrm{du}$ longissimus et latéraux de l'iliocostalis présentent une innervation plurisegmentaire avec chevauchement sur 2 à 4 myotomes, rendant plus délicate l'interprétation EMG de l'atteinte radiculaire [3, 8]. 


\section{MATÉRIEL ET MÉTHODES}

\section{Population étudiée}

Nous avons examiné 75 sujets sains, volontaires ayant donné leur consentement éclairé, en respectant les critères d'exclusion suivants :

- rachialgie évolutive ou chronique ;

- arthrose ou hernie discale dorso-lombaire;

- pathologie inflammatoire ostéoarticulaire ou neuromusculaire ;

- affection métabolique (diabète, dysthyroïdie, ...) ;

- trouble de l'hémostase.

La population de référence comporte 40 hommes (moyenne d'âge de 37 ans \pm 12 ) et 35 femmes (moyenne d'âge de 38 ans \pm 13 ), avec des âges extrêmes de 19 et 86 ans. L'effectif peut se répartir au travers de 3 classes d'âge. La première de 20 à 33 ans concerne 21 sujets pour T8 et 35 sujets pour L5 ; la deuxième de 34 à 49 ans inclut, tant pour T8 que pour L5, 18 sujets ; enfin, pour les plus de 50 ans, il y a 15 sujets pour T8 et 17 sujets pour L5. Cette population est soumise à une étude EMG des faisceaux musculaires paravertébraux du multifidus dorsal en T8 $(n=54)$ et lombo-sacré en L3 $(n=12)$, L4 $(n=12), \mathrm{L} 5(n=70)$ et S1 $(n=12)$.

Au niveau lombaire, le choix préférentiel de L5 dans le recrutement de l'effectif repose sur l'incidence habituellement élevée des radiculopathies à topographie L5. Pour chaque niveau segmentaire, 20 potentiels d'unité motrice (PUMs) différents ont été étudiés. Les paramètres électrophysiologiques classiquement décrits $[1,7$, 15, 17, 19] pour caractériser les PUMs (amplitude, durée, caractère polyphasique) sont analysés.

\section{Description de la technique}

L'installation confortable du patient en procubitus, avec au besoin un coussin sous le ventre pour effacer la lordose lombaire, constitue l'étape préliminaire indispensable à un examen de bonne qualité.

L'étude EMG de la musculature paravertébrale dorso-lombaire est inspirée de la technique du Paraspinal Mapping décrite par Haig et al. [9, 10, 11, 12, 18] (Fig. 1A).

Chaque site d'insertion de l'aiguille EMG (2 à 3 par niveau segmentaire) est abordé avec une angulation de 45 à $60^{\circ}$ sur l'horizontale, en direction médiale et sur une profondeur de 2,5 à $3 \mathrm{~cm}$, permettant d'explorer 3 à 5 plages musculaires par site $[2,9]$.
$\mathbf{A}$

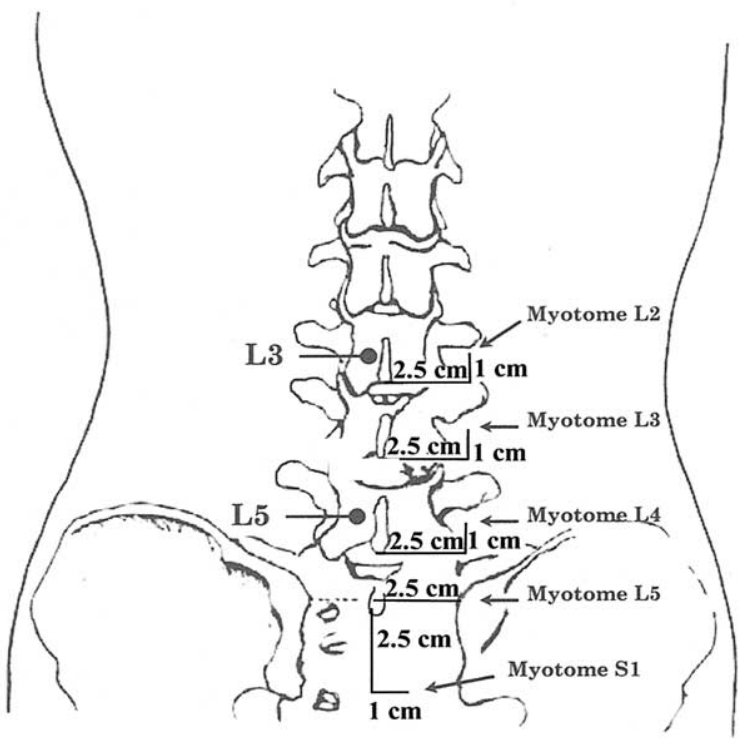

B

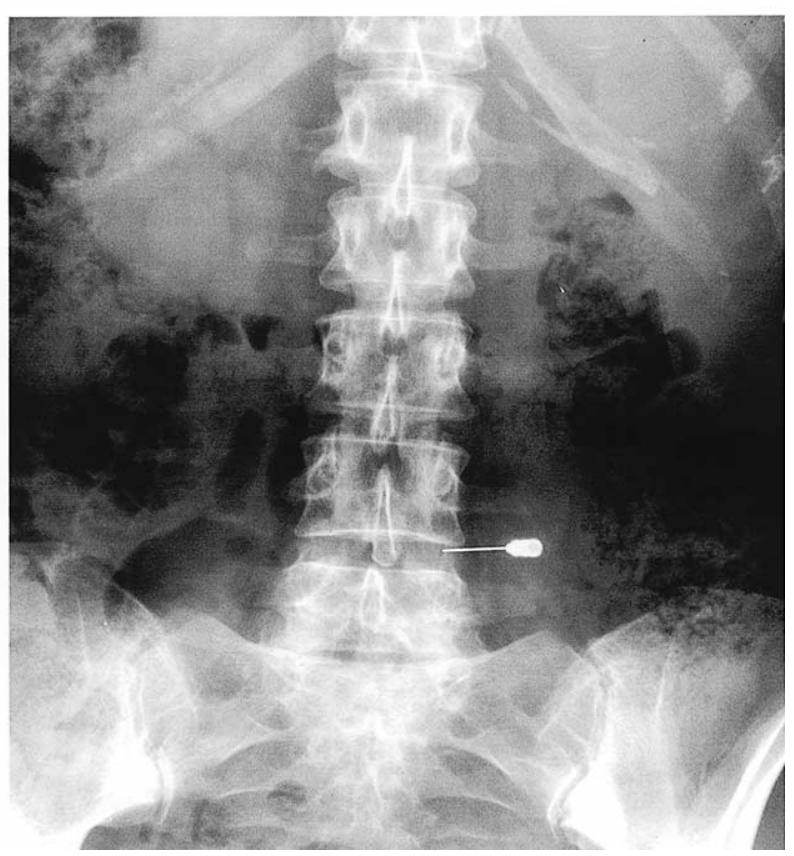

Fig. 1. Illustration de la technique électromyographique (EMG) d'exploration du multifidus lombaire. (A) Sites d'insertion de l'aiguille EMG en fonction du myotome étudié (d'après Haig [10]). (B) Image radiologique de l'insertion de l'aiguille EMG en paravertébral L4-L5 pour l'étude du myotome L3 gauche. 
Les repères d'insertion pour les myotomes T8, L3 et L4 se projettent $2,5 \mathrm{~cm}$ latéralement et $1 \mathrm{~cm}$ en direction rostrale par rapport à l'épineuse sous-jacente au niveau segmentaire étudié. Pour L5, le repère se situe à $2,5 \mathrm{~cm}$ en dehors du centre d'une ligne virtuelle joignant les épines iliaques postéro-supérieures. Le point d'insertion pour $S 1$ se localise $2,5 \mathrm{~cm}$ en-dessous du centre de cet axe et $1 \mathrm{~cm}$ latéralement [8,12] (Fig. 1A). Les apophyses épineuses du niveau segmentaire L4-L5 se projettent habituellement à hauteur du rebord postérieur de la crête iliaque $[8,9,12,18]$.

L'analyse des tracés EMG s'effectue sur un appareil de marque Medtronic, disposant du logiciel Keypoint, au moyen d'électrode-aiguille bipolaire concentrique de $0,07 \mathrm{~mm}^{2}$ de surface d'enregistrement. Les filtres de fréquences sont réglés pour une limite inférieure de bande passante à $5 \mathrm{~Hz}$ et une limite supérieure à $10 \mathrm{kHz}$.

Dans un premier temps, nous recherchons toute forme d'activité spontanée de repos (potentiel de fibrillation, potentiel lent de dénervation, fasciculation). Nous examinons ensuite systématiquement en mode multi-MUP (Motor Unit Potential) 20 PUMs distincts, obtenus lors de l'extension contrôlée à faible intensité (5 à $30 \%$ de la force isométrique maximale) du rachis ou du membre inférieur homolatéral.

\section{Analyse statistique}

Le coefficient de variabilité $(C V)$ apprécie en pourcentage la reproductibilité des mesures $x_{1}$ et $x_{2}$ enregistrées lors de deux tests successifs. Il se calcule par la formule :

$$
C V=S D / m 100 \%
$$

où l'écart type

$$
S D=\sqrt{\sum\left(x_{1}-x_{2}\right)^{2} / 2 n}
$$

et la moyenne $m$ est obtenue sur $2 n$ (effectifs $n_{1}+n_{2}$ ).

Pour comparer les différents paramètres des PUMs en fonction du sexe et de l'âge, nous calculons le coefficient de corrélation de Bravais-Pearson ( $r$ ) et la probabilité de dépassement ( $p$-value) selon un test statistique $t$ de Student au seuil d'incertitude $\alpha=5 \%$.

La confrontation des paramètres EMG en L3, L4, L5 et $S 1$ s'appuie sur une analyse statistique de la variance à deux critères (ANOVA-2) par le biais d'un test d'hy- pothèse pour échantillons appariés avec calcul de la $p$-value et d'un test $F$ de Snedecor (niveau d'incertitude $\alpha=5 \%)$.

Les normes définies sur l'ensemble de la population étudiée sont obtenues par la formule :

$$
m \pm Q t(1-\alpha / 2 ; n-1) \sqrt{1+1 / n} \mathrm{SD}
$$

où le quantile

$$
Q t(1-\alpha / 2 ; n-1)
$$

est lu dans la table d'une loi $t$ de Student pour un niveau d'incertitude $\alpha=5 \%$. Cette formule équivaut à $m \pm 1.96 S D$ quand l'effectif $\mathrm{n}$ tend vers l'infini.

Les moyennes des paramètres analysés reflètent pour chaque sujet la moyenne de 20 PUMs distincts.

La représentation graphique des normes sous forme d'aire rectangulaire est obtenue par l'association des limites inférieure et supérieure de la normale pour les paramètres amplitude et durée des PUMs (Fig. 2). Nous définissons d'une part des rectangles normatifs relatifs aux valeurs moyennes des paramètres étudiés. Chez un patient, le muscle est considéré pathologique si la valeur moyenne des 20 PUMs collectés se situe en dehors de ce rectangle. D'autre part, nous établissons des rectangles normatifs relatifs aux limites extrêmes de l'ensemble des valeurs individuelles. Pour chaque sujet, les 3 e plus petite et plus grande valeurs mesurées parmi les 20 PUMs sélectionnés sont retenues [16]. La plus petite et la plus grande de ces valeurs définissent sur l'ensemble de notre population les limites extrêmes du paramètre étudié, pour un niveau d'incertitude égal à $10 \%$. En clinique, un muscle est qualifié de pathologique si 3/20 (>10\%) PUMs, définis par une amplitude et une durée, se situent en dehors du rectangle normatif pour les valeurs individuelles.

\section{RÉSULTATS}

\section{Repères palpables}

Afin de valider la qualité méthodologique de l'étude, nous avons vérifié le positionnement correct de l'aiguille EMG lors d'un contrôle radiologique auprès de 24 sujets témoins ne participant pas à l'étude EMG proprement dite ( 12 femmes et 12 hommes) (Fig. 1B). Le niveau segmentaire L3, L4, L5 ou S1 a été attribué par tirage au sort préalable et 3 sujets témoins ont été 


\section{Normes pour T8}

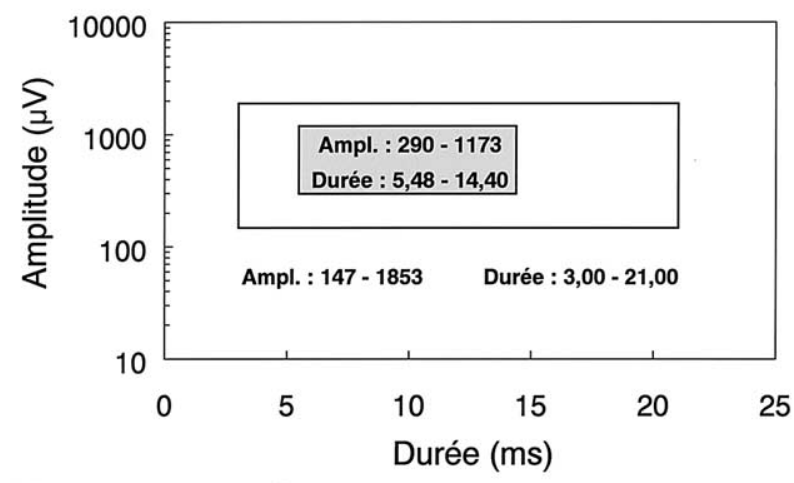

Normes pour L5

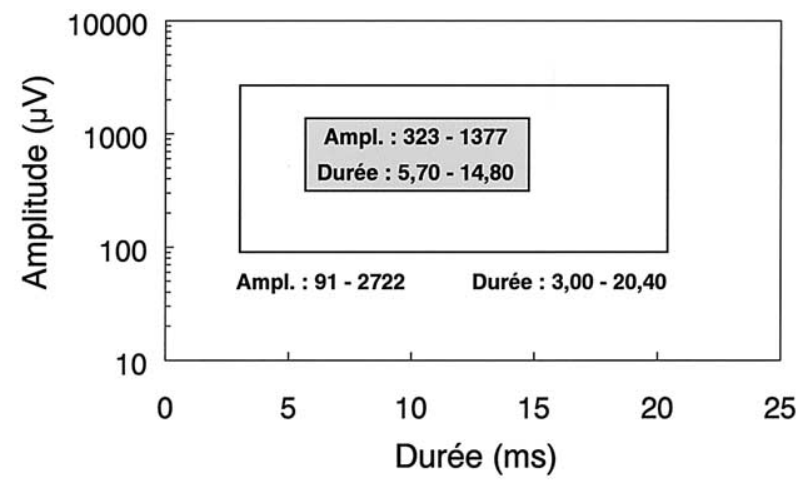

Fig. 2. Valeurs de référence en T8 $(n=1.080)$ et $L 5(n=1.400)$ représentées par des rectangles normatifs définis par les limites supérieures et inférieures de la normale de l'amplitude $(\mu \mathrm{V})$ et de la durée (ms) des potentiels d'unité motrice (PUMs). Les rectangles gris sont calculés à partir des valeurs moyennes $\pm 2 \mathrm{SD}$. Les rectangles blancs sont établis à partir des limites extrêmes de l'ensemble des valeurs individuelles. Les limites extrêmes sont définies par la plus grande et la plus petite valeurs de l'ensemble des 3èmes plus grandes (P17) et plus petites (P3) amplitudes et durées mesurées parmi les 20 PUMs enregistrés chez chaque sujet. recrutés pour chaque niveau. L'aiguille EMG est d'abord insérée sur base des repères anatomiques décrits par Haig ; ensuite un cliché radiographique de face permet d'apprécier objectivement le positionnement de l'aiguille.

Pour les myotomes L3 et L5, 100\% des insertions sont correctes. En L4, on relève 66,6\% d'insertion correcte, $16,6 \%$ d'insertion incorrecte et $16,6 \%$ d'insertion discutable dans le sens où le niveau segmentaire d'insertion est correct sans toutefois pouvoir assurer le positionnement adéquat de l'aiguille EMG au sein du myotome recherché compte tenu de la disposition transversale des muscles rachidiens comme détaillée ci-dessus. En S1, 83,3\% des insertions sont correctes ; la position incorrecte étant associée à une anomalie transitionnelle de la charnière lombo-sacrée. En somme, pour les différents niveaux étudiés (L3 à S1), l'insertion de l'aiguille s'est avérée inadéquate chez $2 / 12$ et discutable pour $1 / 12$ des sujets étudiés.

\section{Activité spontanée de dénervation}

Aucun des 75 sujets sains examinés n'a démontré la moindre activité de repos. Même pour la catégorie d'âge supérieur à 50 ans, aucun potentiel de fibrillation ou fasciculation ou potentiel lent de dénervation n'a été enregistré.

\section{Analyse quantitative des PUMs}

La reproductibilité de la technique EMG en mode multi-MUP est appréciée sur 10 sujets sains (moyenne d'âge : 46 ans \pm 15 ; extrêmes : 20-62 ans) en réalisant 2 tests consécutifs à une semaine d'intervalle (Tableau 1). Les deux enregistrements sont réalisés dans des conditions strictement similaires. Notre analyse porte sur les paramètres quantitatifs des PUMs obtenus par la méthode multi-MUP $[2,16,17]$. Le calcul du coefficient de variabilité est donné pour l'amplitude, la durée et le pourcentage de PUMs polyphasiques. Les valeurs sont reprises dans le Tableau 1 . Nous obtenons

Tableau 1. Reproductibilité des résultats de l'analyse en mode multi-MUP (Motor Unit Potential) par le calcul du coefficient de variabilité (CV).

\begin{tabular}{lccc}
\hline Paramètres & $m \pm S D$ Test 1 & $m \pm S D$ Test 2 & $C V(\%)$ \\
\hline Amplitude $(\mu \mathrm{V})$ & $725 \pm 191$ & $701 \pm 190$ & 12 \\
Durée $(\mathrm{ms})$ & $11,9 \pm 1,6$ & $11,4 \pm 1,9$ & 10,1 \\
Polyphasique $(\%)$ & $6,0 \pm 5,7$ & $7,0 \pm 3,5$ & 64,4 \\
\hline
\end{tabular}

$m=$ moyenne des 20 potentiels d'unité motrice enregistrés chez chacun des 10 sujets contrôles. 
Tableau 2. Influence du sexe sur les potentiels d'unité motrice en T8 $(n=54)$ et en L5 $(n=70)$.

\begin{tabular}{llll}
\hline Paramètres en T8 & $\begin{array}{c}\text { Femmes } \\
(n=29)\end{array}$ & $\begin{array}{c}\text { Hommes } \\
(n=25)\end{array}$ & $p$ (t de Student) \\
\hline Amplitude $(\mu \mathrm{V})$ & $581 \pm 197$ & $662 \pm 251$ & 0,167 \\
Durée $(\mathrm{ms})$ & $9,7 \pm 2,1$ & $10,3 \pm 2,4$ & 0,372 \\
\hline Paramètres en L5 & Femmes & Hommes & $p$ (t de Student) \\
& $(n=33)$ & $(n=37)$ & 0,741 \\
\hline Amplitude $(\mu \mathrm{V})$ & $696 \pm 257$ & $727 \pm 287$ & 0,869 \\
Durée $(\mathrm{ms})$ & $10,3 \pm 1,9$ & $10,2 \pm 2,6$ & \\
\hline
\end{tabular}

Les moyennes des paramètres analysés reflètent pour chaque sujet la moyenne de 20 potentiels d'unité motrice.

des CV entre les deux tests de $12 \%$ pour l'amplitude et de 10,1\% pour la durée. Par contre, une variabilité de $64 \%$ est observée pour la proportion de PUMs polyphasiques.

Nous avons recherché l'influence du sexe sur les paramètres EMG recueillis pour les niveaux segmentaires T8 et L5. L'amplitude et la durée des PUMs ainsi que le pourcentage de PUMs polyphasiques des 35 femmes et 40 hommes sont moyennés (Tableau 2). Aucune différence significative n'est relevée entre les deux sexes pour les paramètres étudiés (Tableau 2).

La relation entre l'âge des 75 sujets et les différents paramètres analysés est établie par le calcul du coefficient de corrélation. Nous constatons une différence significative uniquement pour la durée des PUMs en

Tableau 3. Influence de l'âge sur les potentiels d'unité motrice en T8 $(n=54)$ et en L5 $(n=70)$.

\begin{tabular}{lll}
\hline Paramètres en T8 & $\begin{array}{l}\hat{A g e} \\
r\end{array}$ & $p$ (t de student) \\
\hline Amplitude $(\mu \mathrm{V})$ & 0,168 & 0,224 \\
Durée $(\mathrm{ms})$ & 0,270 & 0,049 \\
\hline Paramètres en $L 5$ & $\hat{A g e}$ & $p$ (t de student) \\
& $r$ & \\
\hline Amplitude $(\mu \mathrm{V})$ & 0,007 & 0,956 \\
Durée $(\mathrm{ms})$ & 0,312 & 0,009 \\
\hline
\end{tabular}

Les moyennes des paramètres analysés reflètent pour chaque sujet la moyenne de 20 potentiels d'unité motrice. ( $r$ = coefficient de corrélation de Bravais-Pearson).
T8 $(p=0,049$ et $r=0,270)$ et L5 $(p=0,009$ et $r=0,312$ ) (Tableau 3).

Enfin, nous avons comparé les résultats des niveaux segmentaires L3, L4, L5 et S1 obtenus sur un échantillonnage de 12 sujets témoins (parité de sexe et moyenne d'âge de 44 ans \pm 15 ). Les paramètres d'amplitude et de durée des PUMs sont moyennés pour chaque niveau segmentaire (Tableau 4). Aucune différence significative n'est notée entre les différents niveaux segmentaires (Tableau 4).

Les normes sont établies sur l'ensemble de la population étudiée en T8 (1080 PUMs) et en L5 (1400 PUMs). Nous définissons un intervalle de référence pour les paramètres d'amplitude et de durée des PUMs par le calcul de la moyenne \pm 2SD (Tableau 5). La Fig. 2 illustre les rectangles normatifs construits à partir des valeurs moyennes et individuelles. La transformation logarithmique de la variable amplitude permet de normaliser une distribution dissymétrique à droite.

\section{DISCUSSION}

La musculature paraspinale est une masse musculaire complexe tant du point de vue de ses rapports anatomiques que de son innervation. L'implication de plusieurs racines nerveuses au niveau des muscles longissimus et iliocostalis rend difficile la localisation d'une atteinte monoradiculaire lors de l'exploration EMG des ces muscles. Par contre, le multifidus lombaire offre par son innervation monosegmentaire une cible EMG privilé-

Tableau 4. Analyse quantitative des potentiels d'unité motrice : comparaison des niveaux segmentaires L3, L4, L5, S1.

\begin{tabular}{llllll}
\hline Paramètres & $L 3(n=12)$ & $L 4(n=12)$ & $L 5(n=12)$ & $S 1(n=12)$ & $p($ Anova-2) \\
\hline Amplitude $(\mu \mathrm{V})$ & $687 \pm 228$ & $797 \pm 338$ & $723 \pm 167$ & $872 \pm 339$ & 0,353 \\
Durée $(\mathrm{ms})$ & $12,5 \pm 1,9$ & $12,6 \pm 1,8$ & $11,6 \pm 2,2$ & $11,2 \pm 2,4$ & 0,099 \\
\hline
\end{tabular}


Tableau 5. Intervalles de référence (limite inférieure-limite supérieure), des paramètres quantitatifs des potentiels d'unité motrice en T8 et L5, établis par le calcul de la valeur moyenne $\pm 2 S D$.

\begin{tabular}{lcc}
\hline Paramètres & $T 8$ & L5 \\
\hline Amplitude $(\mu \mathrm{V})$ & $290-1173$ & $323-1377$ \\
Log. Amplitude $(\mu \mathrm{V})$ & $5,7-7,1$ & $5,8-7,2$ \\
Durée $(\mathrm{ms})$ & $5,5-14,4$ & $5,7-14,8$ \\
\hline
\end{tabular}

giée dans la recherche topographique d'une atteinte radiculaire.

L'étude EMG des muscles paravertébraux lombaires par électrode-aiguille devrait être dès lors valorisée notamment lorsqu'une radiculalgie clinique est confirmée par la mise en évidence d'anomalies électrophysiologiques périphériques.

La difficulté principale de l'exploration EMG des muscles paraspinaux réside dans le repérage exact du niveau segmentaire à étudier. En préambule à l'étude EMG proprement dite, la confrontation radiologique des repères palpables a été utilisée pour apprécier la fiabilité de la technique utilisée. Chez 24 sujets témoins, nous avons vérifié le positionnement correct de l'aiguille EMG par un cliché radiologique (Fig. 1B). Au total, nous enregistrons un taux d'erreur de $8,3 \%$ et un test discutable pour $4,2 \%$ des sujets étudiés. Comparativement, les résultats de l'étude cadavérique de Haig et al. (où la spécificité topographique des faisceaux musculaires est révélée par injection de colorant au latex et dissection ultérieure) donnent un taux de précision variant de 81 et 97\% [9]. La présence d'une anomalie transitionnelle de la charnière lombo-sacrée (c'est le cas pour l'un de nos résultats erronés) peut compliquer la prise des repères palpables.

Comme pour les autres muscles, l'étude EMG des paraspinaux comporte d'abord l'analyse de l'activité électrique dans les conditions de repos musculaire. L'absence d'activité spontanée de repos au niveau de la musculature para-vertébrale sur l'ensemble de notre population de référence était logiquement attendue. Il importe de ne pas confondre une hypothétique activité de repos avec un manque de relaxation musculaire, une activité d'insertion musculaire ou des potentiels de plaque motrice voire des potentiels fusimoteurs $[7,15]$. Ces sources d'erreurs assez fréquentes seront évitées par l'installation adéquate des sujets et par le positionnement optimal et minutieux de l'aiguille EMG [7]. Les précautions méthodologiques adoptées dans ce travail pourraient rendre compte des différences de résultats entre notre série et les études de Nardin et al. et Date et al. qui rapportent respectivement $40 \%$ et $14,5 \%$ d'activité de repos au niveau des muscles paravertébraux d'une population asymptomatique $[6,16]$. Les phénomènes involutifs ostéoarticulaires et neuromusculaires locaux (souvent méconnus du patient) dont la fréquence augmente significativement avec l'âge pourraient influencer également les résultats obtenus par ces auteurs $[4,5,6,7,19]$. Cependant, dans notre étude, bien que la population étudiée présente une moyenne d'âge relativement jeune ( $37 \pm 12$ ans pour les hommes et $38 \pm 13$ ans pour les femmes), pratiquement la moitié de l'effectif est âgée de plus de 34 ans et $25 \%$ des sujets ont plus de 50 ans. Enfin, les critères d'exclusion imposés dans ce travail ont peut-être permis d'exclure davantage de patients asymptomatiques, âgés ou non, que dans d'autres études.

La reproductibilité des résultats de l'analyse des PUMs en mode multi-MUP a été vérifiée par le calcul du coefficient de variabilité entre deux tests successifs réalisés à une semaine d'intervalle chez 10 sujets sains (Tableau 1). La reproductibilité est satisfaisante pour la durée des PUMs $(C V=10,1 \%)$, or on sait que ce paramètre est un des plus sensibles dans la détection d'une pathologie musculaire même débutante. Le $C V$ excède discrètement le seuil de $10 \%$ pour l'amplitude des PUMs, ce qui ne doit certainement pas remettre en cause l'utilité de ce paramètre dans la détection d'une pathologie radiculaire chronique. Seul le pourcentage des PUMs polyphasiques montre une importante variabilité entre les deux tests, modulant en conséquence l'interprétation des résultats pathologiques. C'est pourquoi il n'apparaît pas dans l'exposé des résultats.

La comparaison des paramètres électrophysiologiques obtenus par l'analyse des PUMs en mode multiMUP aux niveau T8 et L 5 révèle l'absence de différence significative entre les femmes et les hommes ( $\mathrm{Ta}-$ bleau 2). Aucune corrélation significative de ces mêmes paramètres avec l'âge n'est davantage relevée (Tableau 3). Même si la durée des PUMs augmente significativement avec l'âge en T8 et L5, les corrélations sont faibles et sans implication clinique. En effet, le coefficient de détermination $r^{2}$, expliquant la fluctuation d'une variable par une autre, reste inférieur à $9 \%$. Ces résultats sont conformes à ceux de la littérature $[2,17]$. La comparaison des différents paramètres étudiés entre les différents niveaux segmentaires L3, L4, L5 et S1 ne révèle également aucune différence significative (Tableau 4).

L'absence de relation entre les paramètres d'analyse quantitative des PUMs et l'âge ou le sexe, nous permet 
de disposer d'un effectif statistiquement conséquent pour les myotomes T8 (1080 PUMs) et L5 (1400 PUMs), pour établir des valeurs normatives (Fig. 2, Tableau 5). Dans le but d'améliorer la sensibilité de détection des cas pathologiques, la méthode des rectangles normatifs établis par combinaison des paramètres d'amplitude et de durée des PUMs a été retenue [16, 17] (Fig. 2). En effet, il a été démontré que les limites extrêmes définies à partir des valeurs individuelles sont aussi sensibles que celles établies par les valeurs moyennes pour détecter une neuropathie, alors qu'elles s'avèrent plus performantes en cas de myopathie [16]. Un avantage supplémentaire du principe des limites extrêmes établies sur les valeurs individuelles est de ne pas nécessiter l'enregistrement de 20 PUMs, puisque cette technique est jugée positive dès que 3 PUMs sont hors normes, ce qui implique moins de douleur pour le patient et un gain de temps pour le médecin.

Dans la littérature, seule l'étude de Barkhaus et al. propose des valeurs chiffrées de l'analyse quantitative des PUMs au niveau de la musculature paravertébrale [1]. Ce travail ne comporte cependant qu'un petit nombre de sujets sains, âgés de 26 à 45 ans $(n=11$; multifidus dorsal : 363 PUMs ; multifidus lombaire : 369 PUMs). Il differe de notre étude car l'objectif n'était pas de focaliser l'analyse sur un myotome segmentaire précis mais de comparer globalement les niveaux cervical, dorsal et lombaire.

En pratique, outre la confirmation diagnostique des radiculopathies, cette base de données de référence s'avérera utile pour l'exploration des atteintes proximales plexuelles ou tronculaires, où on s'attend à retrouver une musculature paravertébrale indemne de toute atteinte neurogène $[7,16,19]$. À l'inverse, les pathologies de la corne antérieure et les pathologies myogènes s'expriment souvent préférentiellement et/ou précocement au niveau de la musculature paravertébrale.

L'exploration EMG des muscles paraspinaux n'est pas dénuée de complication potentielle, néanmoins jamais rencontrée dans ce travail (hématome intramusculaire, exceptionnelle brèche péridurale, risque infectieux minimisé par le respect des règles d'asepsie), et peut s'avérer techniquement délicate dans certaines circonstances. Parmi celles-ci, citons la présence d'une hyperlordose lombaire très prononcée, ou d'un panicule adipeux très épais, ou encore d'une contracture musculaire importante, préexistante à l'exploration EMG, d'origine réflexe en relation avec le syndrome rachidien douloureux. Mais, l'écueil principal de cette pratique EMG se situe dans le délai de réalisation de cette étude $[8,19]$. En effet, puisque les mécanismes de dénervation suivent un cheminement à point de départ proximal, une activité spontanée de dénervation peut être observée dans un délai de 7 à 10 jours au niveau de la musculature paraspinale [12] alors qu'il faut souvent attendre 3 voire 5 à 6 semaines au niveau des muscles périphériques $[8,19]$. Or généralement, seul un nombre limité de patients bénéficient d'une exploration EMG avant 2 à 3 semaines d'évolution symptomatique, sauf évidemment pour les cas de parésie invalidante. Ainsi, l'activité de repos au niveau du multifidus lombaire peut avoir disparu sans que l'altération des paramètres quantitatifs des PUMs ne soit déjà objectivée.

En définitive, l'étude EMG du multifidus lombaire est une donnée intéressante dans la mise au point des radiculopathies lombo-sacrées grâce à sa précision topographique. Les valeurs normatives présentées dans ce travail, à partir de l'examen de 75 sujets sains, sont donc potentiellement utiles pour l'exploration de la pathologie radiculaire, mais aussi dans certains types de myopathie ou d'atteinte de la corne antérieure. Enfin, la reproductibilité des résultats concernant la durée et l'amplitude des PUMs et les données de la confrontation radiologique des repères palpables soulignent la fiabilité de cette méthodologie.

\section{REMERCIEMENTS}

Les auteurs remercient le Professeur A. ALBERT pour sa contribution à l'analyse statistique des paramètres EMG.

\section{RÉFÉRENCES}

1 Barkhaus P, Periquet I, Nandedkar S. Quantitative motor unit action potential analysis in paraspinal muscles. Muscle Nerve 1997;20:373-5.

2 Bischoff C, Stalberg E, Falck B, Edeboleeg-Olofsson K. Reference values of motor unit action potentials obtained with multi-MUAP analysis. Muscle Nerve 1994;17:842-51.

3 Bogduk N, Wilson A, Tynan W. The human lumbar dorsal rami. J Anat 1982;134(2):383-97.

4 Boulu P, Benoist M. Recent data on the pathophysiology of nerve root compression and pain. Rev Rhum (Engl. Ed.) 1996;63(5):358-63.

5 Czyrny J, Lawrence J. The importance of paraspinal muscle EMG in cervical and lumbosacral radiculopathy: review of 100 cases. Electromyogr Clin Neurophysiol 1996;36:503-8.

6 Date E, Mar E, Bugola M, Teraoka J. The prevalence of lumbar paraspinal spontaneous activity in asymptomatic subjects. Muscle Nerve 1996;19:350-4. 
7 Dumitru D. Physiologic basis of potentials recorded in electromyography. Muscle Nerve 2000;23:1667-85.

8 Frank L, Schneider D, Zuhosky J. Anatomic and technical considerations in needle electromyography of the lumbar spine. Phys Red Rehabil Clin N Am 1998;9(4):795-814.

9 Haig A, Moffraid M, Haugh L, Pope M. A technique for needle localisation in paraspinal muscles with cadaveric confirmation. Muscle Nerve 1991;14:521-6.

10 Haig A, Talley C, Grabler L, Le Breck D. Paraspinal Mapping: quantified needle electromyography in lumbar radiculopathy. Muscle Nerve 1993;16:477-84.

11 Haig A. Clinical experience with paraspinal mapping. I. Neurophysiology of the paraspinal muscles in various spinal disorders. Arch Phys Red Rehabil 1997;78:1177-84.

12 Haig A. Clinical experience with paraspinal mapping. II. Simplified technique that eliminates three-fourths of needle insertions. Arch Phys Med Rehabil 1997;78:1185-9.

13 Johnson EW, Melvin JL. Value of electromyography in lumbar radiculopathy. Arch Phys Med Rehabil 1971;52:239-43.
14 Mac Intosh J, Bogduk N, Munro R. The morphology of the human lumbar multifidus. Clinical Biomechanics 1986;1: 196-204.

15 Nardin R, Raynor E, Rutkove S. Fibrillations in lumbosacral paraspinal muscles of normal subjects. Muscle Nerve 1998;21: 1347-9.

16 Stalberg E, Bischoff C, Falck B. Outliers, a way to detect abnormality in quantitative EMG. Muscle Nerve 1994;17: 392-9.

17 Stalberg E, Falck B, Sonoo M, Stalberg S, Aström M. MultiMUP EMG analysis - a two year experience in daily clinical work. Electroencephalogr Clin Neurophysiol 1995;97: 145-54.

18 Stein J, Baker E, Pine Z. Medial paraspinal muscle electromyography: Techniques of examination. Arch Phys Red Rehabil 1993;74:497-500.

19 Wilbourn A, Aminoff M. AAEM Min. 32: The electrodiagnostic examination in patients with radiculopathies. Muscle Nerve 1998;21:1612-31. 